\title{
Expression and phylogenetic analysis of the zic gene family in the evolution and development of metazoans
}

\author{
Michael J Layden, Néva P Meyer, Kevin Pang, Elaine C Seaver, Mark Q Martindale*
}

\begin{abstract}
Background: zic genes are members of the gli/glis/nkl/zic super-family of $\mathrm{C} 2 \mathrm{H} 2$ zinc finger (ZF) transcription factors. Homologs of the zic family have been implicated in patterning neural and mesodermal tissues in bilaterians. Prior to this study, the origin of the metazoan zic gene family was unknown and expression of zic gene homologs during the development of early branching metazoans had not been investigated.

Results: Phylogenetic analyses of novel zic candidate genes identified a definitive zic homolog in the placozoan Trichoplax adhaerens, two gli/glis/nkl-like genes in the ctenophore Mnemiopsis leidyi, confirmed the presence of three gli/glis/nkl-like genes in Porifera, and confirmed the five previously identified zic genes in the cnidarian Nematostella vectensis. In the cnidarian $\mathrm{N}$. vectensis, zic homologs are expressed in ectoderm and the gastrodermis (a bifunctional endomesoderm), in presumptive and developing tentacles, and in oral and sensory apical tuft ectoderm. The Capitella teleta zic homolog (Ct-zic) is detectable in a subset of the developing nervous system, the foregut, and the mesoderm associated with the segmentally repeated chaetae. Lastly, expression of gli and glis homologs in Mnemiopsis. leidyi is detected exclusively in neural cells in floor of the apical organ.

Conclusions: Based on our analyses, we propose that the zic gene family arose in the common ancestor of the Placozoa, Cnidaria and Bilateria from a gli/glis/nk/like gene and that both ZOC and ZF-NC domains evolved prior to cnidarian-bilaterian divergence. We also conclude that zic expression in neural ectoderm and developing neurons is pervasive throughout the Metazoa and likely evolved from neural expression of an ancestral gli/glis/nkl/zic gene. zic expression in bilaterian mesoderm may be related to the expression in the gastrodermis of a cnidarian-

bilaterian common ancestor.
\end{abstract}

\section{Background}

The zic genes form a sub-family of the gli/glis/nkl/zic transcription factor super-family, which is characterized by the presence of five tandem $\mathrm{C} 2 \mathrm{H} 2$ zinc finger (ZF) DNA binding domains [1-3]. Two key features distinguish the Zic sub-family proteins from the Gli, Glis and Nkl sub-family proteins. Most notably, the number of amino acids between the two cysteine residues in the first $\mathrm{C} 2 \mathrm{H} 2$ zinc finger is increased (Additional File 1) [1]. Secondly, many zic genes contain two additional domains positioned N-terminal to the ZF domains, the Zic1-3 odd-paired conserved (ZOC) domain and the ZF-NC domain (Figure 1A-C) [1-4]. Function has been assigned

\footnotetext{
* Correspondence: mamartin@hawaii.edu

Pacific Biosciences Research Center, Kewalo Marine Laboratory, University of Hawaii, Manoa, 41 Ahui St Honolulu, HI 96813, USA
}

to the ZOC domain [4], while the ZF-NC domain has only been identified by sequence conservation.

Bilaterian and cnidarian zic genes likely arose from a single ancestral gene that radiated independently in both lineages. All characterized bilaterian zic genes contain a conserved intron between the third and fourth ZF domains that is not present elsewhere in the metazoans [1]. Lineage specific $z i c$ gene radiation and $100 \%$ conservation of this common intron in all bilaterian zics support a single urbilaterian zic gene. Furthermore, in the basal deuterostome echinoderm Strongylocentrotus purpuratus and in most protostome lineages only a single zic gene has been identified [1]. An exception is the platyhelminthes lineage, which has been shown to possess two zic paralogs (see DjaZicA, B Figure 2) [1]. zic gene number expanded via tandem and chromosomal duplication
C Biomed Central

C 2010 Layden et al; licensee BioMed Central Ltd. This is an Open Access article distributed under the terms of the Creative Commons Attribution License (http://creativecommons.org/licenses/by/2.0), which permits unrestricted use, distribution, and reproduction in any medium, provided the original work is properly cited. 


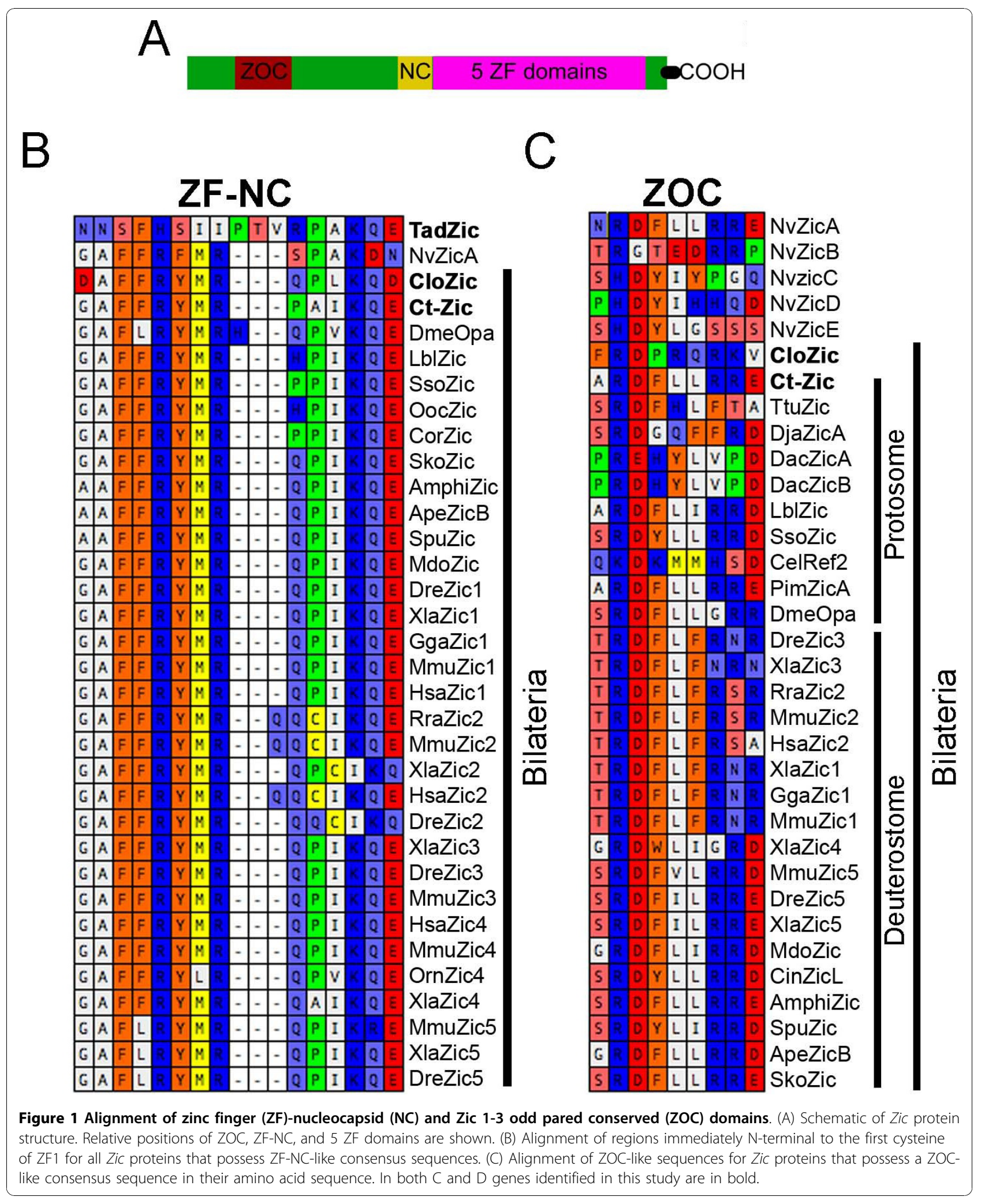




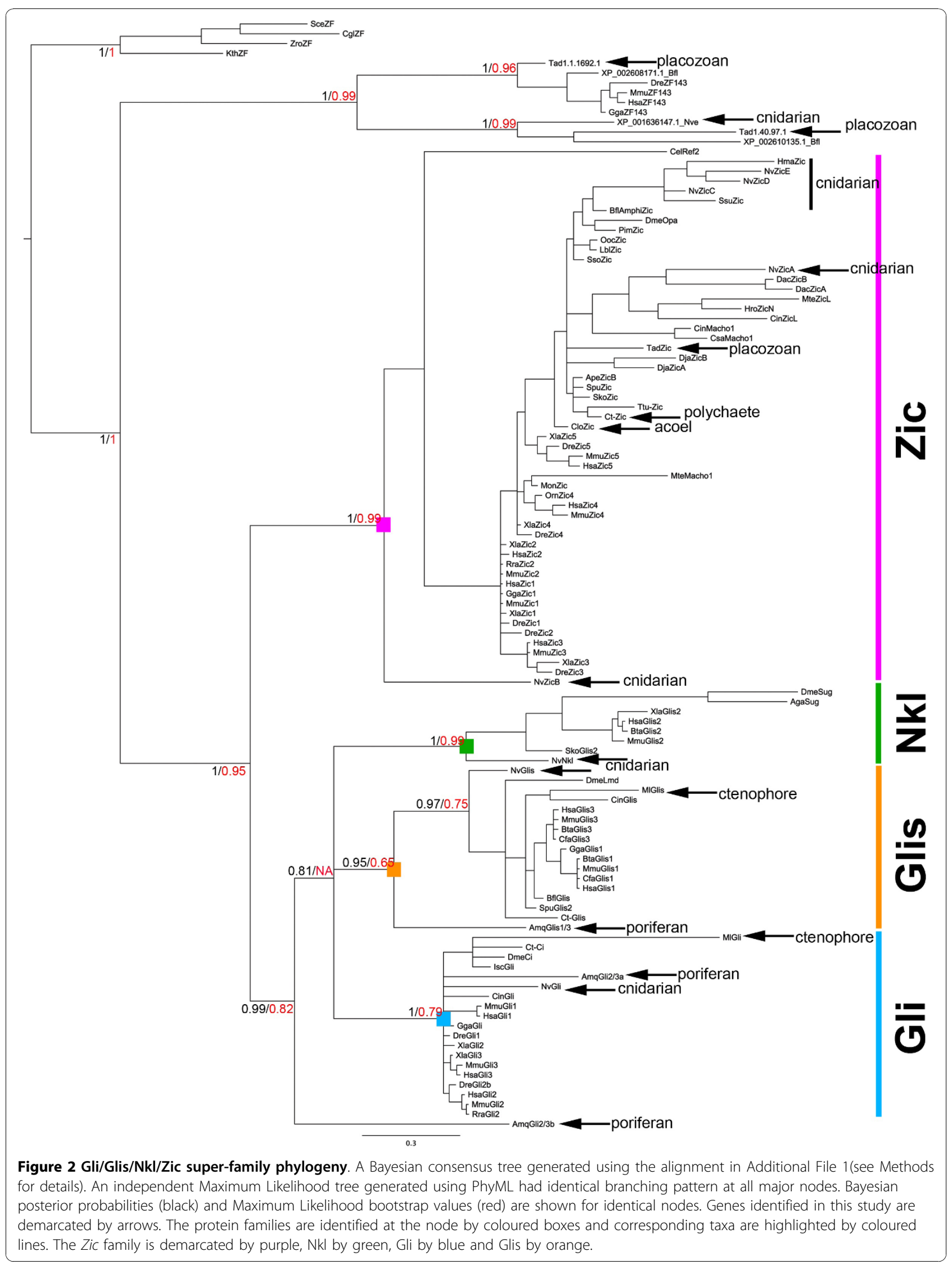


in the chordate lineage from a single ancestral gene in the basal cephalochordate to two in urochordates and five in vertebrates [1-3].

The cnidarians are the only basal metazoan known to possess zic gene homologs. A single zic gene was identified in polymerase chain reaction (PCR) surveys of Hydra vulgaris [5] and the jellyfish Scolionema suvaense [1]. Nematostella vectensis possesses five zic genes, of which four are arranged tandemly on the same genomic scaffold [1], suggesting tandem duplication from a single ancestral gene [1]. Recent sequencing of the Amphimedon queenslandica sponge genome identified one putative ancestral gli/glis/nkl gene, one glis-like gene and one gli-like gene [6]. However, no zic homolog was identified [6]. zic homologs have not been verified in placozoans and ctenophores.

Expression of zic homologs during bilaterian development suggests that the urbilaterian zic gene was expressed in neural and mesodermal domains. zic expression in protostomes (Ecdysozoa and Lophotrochozoa) has been characterized in Drosophila melanogaster, Caenorhabditis elegans and Tubifex tubifex. The fruit fly D. melanogaster zic (odd-paired; opa) is expressed in all ectodermal and mesodermal precursors in the presumptive segmented region of the embryo $[7,8]$. At later stages opa is expressed in the neural ectoderm [9] and in a subset of visceral mesoderm around the midgut [8]. In the nematode C. elegans, the zic homolog, ref-2, is expressed in a subset of neural precursors [10,11]. In the lophotrochozoan oligochaete T. tubifex, Ttuzic expression is observed in the mesodermal m-blast cells and germ bands, mesoderm associated with chaetal sacs, anterior ectoderm and the developing brain [12]. In summary, protostome zic genes are expressed in a subset of neural-ectoderm and a sub-set of mesoderm. However, the structures expressing zic within a particular germ layer vary between species (subset of mesodermal precursors in T. tubifex versus broad mesodermal precursor in D. melanogaster). Moreover, expression even within similar tissues (for example, neural) varies in both timing and location (anterior brain in T. tubifex versus ventral nerve cord (VNC) in D. melanogaster).

In deuterostomes, zic expression and function have been best characterized in the chordate lineage. The five vertebrate $z i c$ gene expression patterns show extensive overlap during development. In the mouse, zebrafish and Xenopus embryos, zic is expressed in presumptive ectoderm at or just prior to gastrulation [13]. The ectodermal expression resolves to the neural plate and is ultimately restricted to the dorsal neural tube and the lateral boundary of the neural plate where neural crest cells will form [2,3]. In addition, vertebrate zic genes are expressed in the dorsal somitic mesoderm and MmuZic1 and MmuZic3 are expressed in the developing retina [13]. In ascidians, two zic genes have been identified (macho-1 and $z i c L / N$ ), which display similar patterns among the three species examined [14-18]. zicL/N is expressed in presumptive muscle and notochord mesodermal lineages and in neural precursors [14-16]. macho-1 mRNA is localized to mesodermal precursor blastomeres [17,18]. The amphioxus zic gene (amphizic) is detected in the dorsal ectoderm and underlying mesoderm in gastrula, in the mesodermally-derived roof of the anterior archenteron, in the dorsal neural tube and in dorsal-lateral somatic mesoderm in the developing somites [14].

Previously, the only zic gene expression pattern to be characterized in early branching metazoans was in the cnidarian $H$. vulgaris. In these animals zic (hyzic) expression is detected in adult polyps within the dividing i-cells [5], which are stem cells that generate cnidocytes, neurons and other cell types [5]. However, embryonic expression of zic gene homologs has yet to be characterized in cnidarians.

In order to better understand the diverse roles of zic genes and their potential role in the evolution and development of metazoans, we have investigated the origins and expression of the zic gene family in a diverse group of previously unsampled metazoans. Our findings indicate that the zic gene family probably arose from a gli/ glis/nkl-like precursor gene in the common ancestor of Placozoa/Cnidaria/Bilateria, as supported by a definitive zic ortholog in the Trichoplax adhaerens genome and a lack of zic homologs in either of the more basally branching Ctenophora or Porifera. Additionally, we present expression patterns for zic genes during the development of the cnidarian $N$. vectensis, the lophotrochozoan C. teleta and for gli and glis genes in the ctenophore M. leidyi.

\section{Results}

\section{Zic genes evolved prior to the split between Placozoa} and Cnidaria

We identified putative $z i c$ homologs in early branching metazoans by degenerate PCR and genome searches (see Materials and Methods for details). We searched poriferan, ctenophore, and placozoan genomes because no zic sequences had been verified in these metazoan clades. In addition, we searched known fungal genomes and choanoflagellate genomes using Basic Local Alignment Search Tool (BLAST) approaches. In order to identify putative gli/glis/nkl/zic genes, sequences were aligned to previously characterized $\mathrm{gli} / \mathrm{glis} / \mathrm{nkl} / \mathrm{zic}$ genes (see Table 1 for a list of genes and species; Additional File 1 for alignment) and the presence of the five tandem ZF domains was determined. Our search yielded novel candidate $\mathrm{gli} / \mathrm{glis} / \mathrm{nkl} / \mathrm{zic}$ genes in T. adhaerens (three genes), N. vectensis (two), M. leidyi (two), C. teleta (three) and C. longifissura (one). However, we were 
Table 1 List of genes and abbreviations used in this study

\begin{tabular}{|c|c|c|c|}
\hline Phylum & Species & Abbr & Genes used: Accession No./JGI model ID/Sponge ID \\
\hline \multirow[t]{4}{*}{ Fungi } & Zygosaccharomyces rouxii & Zro & ZroZF [XP_002498870] \\
\hline & Saccharomyces cerevisiae & Sce & SceZF [NP_012479] \\
\hline & Candida glabrata & $\mathrm{Cgl}$ & Cgl [XP_447926] \\
\hline & Lancancea thermotolerans & Lth & Lth [XP_002556329] \\
\hline Ctenophora & Mnemiopsis leidyi & Ml & MIGlis [HM265718], MIGLi [Genbank submission number 1388697] \\
\hline Porifera & Amphimedon queenslandica & Amq & AmqGli2/3b [Aqu1.219964], AmqGli2/3a [Aqu1.217717], AmqGlis1/3 [Aqu1.213405] \\
\hline Placozoa & Trichoplax adhaerens & Tad & TadZic[XP_002108473], Tad1.40.97.1 [XP_002118240], Tad1.1.692.1 [XP_002107781] \\
\hline \multirow[t]{3}{*}{ Cnidaria } & Nematostella vectensis & Nv & $\begin{array}{l}\text { NvZicA [AB231867], NvZicB [AB231868], NvZicC [AB231868], NvZicD [AB231868], } \\
\text { NvZicE [AB231868], NvGli [EU162649], NvGlis [XP_001637240], NvNkkl [XP_001636498] } \\
\text { Nvpredprot [XP_001636147.1] }\end{array}$ \\
\hline & Scolionema suvaense & Ssu & SsuZic [AB231883 ] \\
\hline & Hydra magnipapillata & Hma & HmaZic [XP_002166449] \\
\hline Acoelomorpha & Convolutriloba longifissura & $\mathrm{Clo}$ & CloZic [HM235720] \\
\hline Platyhelminthes & Dugesia japonica & Dja & DjaZicA [AB231880], DjaZicB [AB231881] \\
\hline Dicyemida & Dicyema acuticephalum & Dac & DacZicA [AB266039 ], DacZicB [AB266038 ] \\
\hline \multirow[t]{2}{*}{ Annelida } & Tubifex tubifex & Ttu & TtuZic [AB231870 ] \\
\hline & Capitella teleta & $\mathrm{Ct}$ & Ct-Zic [HM235719], Ct-Glis [e_gw1.3.96.1], Ct-Gli [e_gw1.36.42.1] \\
\hline \multirow[t]{4}{*}{ Mollusca } & Loligo bleekeri & Lbl & LblZic [AB231874] \\
\hline & Octopus ocellatus & $\mathrm{Ooc}$ & OocZic [AB231875] \\
\hline & Corbicula sp. & Cor & CorZic [BAE94134] \\
\hline & Spisula soldissima & Sso & SsoZic [BAE94123] \\
\hline Nematoda & Caenorhabditis elegans & $\mathrm{Cel}$ & ref2 [AAM55473] \\
\hline \multirow[t]{4}{*}{ Arthropoda } & Drosophila melanogaster & Dme & opa [NP_524228], ci [NP_524617], Sug [AAS65032], imd [AAF57692] \\
\hline & Ixodes scapularis & Isc & IscGli [XP_002435743] \\
\hline & Pandinus imperator & Pim & PimZic [AB231877] \\
\hline & Anopheles gambiae & Aga & AgaSug [XP_309008] ] \\
\hline \multirow[t]{2}{*}{ Echinodermata } & $\begin{array}{l}\text { Strongylocentrotus } \\
\text { purpuratus }\end{array}$ & Spu & SpuZic [XP_792929], SpuGlis [XP_798511] \\
\hline & Asterina pectinifera & Ape & ApeZic [AB231872] \\
\hline Hemichordata & Saccoglossus kowaleski & Sko & SkoZic [NP_001158430], SkoGlis2 [XP_002738784] \\
\hline \multirow[t]{12}{*}{ Chordata } & Ciona intestinalis & Cin & $\begin{array}{l}\text { CinZicL [NP_001071853], Cinmacho1 [NP_001027958], CinGli [NP_001071951], } \\
\text { CinGlis [NP_001071922] }\end{array}$ \\
\hline & Ciona savignyi & Csa & Csamacho1 [BAB68349] \\
\hline & Halocynthia roretzi & Hro & HroZicN [BAC23063] \\
\hline & Molgula tectiformis & Mte & MteZicL [BAE54350], Mtemacho1 [BAE54349] \\
\hline & Branchiostoma floridae & $B f l$ & BflZic (aka amphiZic) [CAB96573], BflGlis [XP_002611119], Bflpredprot [XP_002610135.1] \\
\hline & Xenopus laevis & Xla & $\begin{array}{l}\text { XlaZic4 [BAF36750], XlaZic2 [AAH82436], XlaGli2 [AAD28180], XlaGlis2 [NP_001082092], } \\
\text { XlaGli3 [NP_001081440.1] XlaZic3 [NP_001081088.1], XlaZic5 [BAA95699.1] }\end{array}$ \\
\hline & Mus musculus & Mmu & $\begin{array}{l}\text { MmuZic5 [NP_075363], MmuZic2 [NP_033600], MmuZic1 [AAH60247], } \\
\text { MmuGlis2 [AF325913], MmuGli1 [BAA85004.1], MmuGli2 [NP_001074594], } \\
\text { MmuGli3 [NP_032156], MmuGlis1 [AAM93156], MmuGlis3 [ZB131654.1] } \\
\text { MmuZic3 [CAM20754.1], MmuZic4 [NP_033602.2], MmuZF143 [EDL16976.1] }\end{array}$ \\
\hline & Rattus rattus & Rra & RraZic2 [NP_001101862], RraGli2 [NP_001100639], \\
\hline & Homo sapiens & Hsa & $\begin{array}{l}\text { HsaZic2 [AAC96325], HsaGli3 [CAB59315], HsaGlis3 [CAH70655], HsaGlis1 [NP_671726], } \\
\text { HsaGli1 [AAM13391.1] HsaGlis2 [NP_115964], HsaZic5 [NP_149123], HsaZic3 [NP_003404], } \\
\text { HsaZic1 [NP_003403.2], HsaZic4 [AAH29507.1] HsaZF143 [CAC17610.1] }\end{array}$ \\
\hline & Canis familiaris & $\mathrm{Cfa}$ & CfaGlis3 [XP_541295], CfaGlis1 [XP_546702.2] \\
\hline & Bos taurus & Bta & BtaGlis1 [XP_615122], BtaGlis2 [DAA15722.1], BtaGlis3 [XP_002689658.1] \\
\hline & Monodelphis domestica & Mdo & MdoZic [XP_001376758] \\
\hline
\end{tabular}


Table 1 List of genes and abbreviations used in this study (Continued)

\begin{tabular}{|c|c|c|}
\hline Danio rerio & Dre & $\begin{array}{l}\text { DreZic4 [NP_001070080], DreGli1 [NP_840081], DreGli2b [NP_001015069], DreZic5 } \\
\text { [AAQ67349.1] } \\
\text { DreZic1 [NP571008.1], DreZic3 [NP001001950.1], DreZic2 [AAG35717.2], DreZf143 } \\
\text { [AA124736.1] }\end{array}$ \\
\hline Gallus gallus & Gga & GgaZic1 [BAB92091], GgaGlis1 [XP_422485], GgaGli1 [P55878], GgaZF143 [XP_426401.2] \\
\hline Ornithorhynchus anatinus & Orn & OrnZic4 [XP_001507901] \\
\hline
\end{tabular}

only able to obtain an $\mathrm{N}$-terminal region containing the ZOC, ZF-NC, and the first 2 ZF domains in a putative zic from the acoel Convolutriloba longifissura (Clozic) by rapid amplification of cDNA ends (RACE) PCR (Additional File 1). No choanoflagellete gli/glis/nkl/zic candidate genes were identified in blast searches (data not shown).

Phylogenetic relationships among candidate genes were determined by performing Bayesian [19] and Maximum Likelihood [20] (Figure 2) analyses using sequences beginning with the first cysteine residue of the first $\mathrm{ZF}$ domain (ZF1) through the last histidine residue in the fifth ZF domain (ZF5) (Additional File 1). Our analyses confirmed previously identified cnidarian zic genes $(N v z i c A-E)$ [1] and suggested that the two novel Nematostella candidate genes were not zic genes but, rather, were members of the glis and $n k l$ clades. Bayesian and Maximum Likelihood analyses grouped NvGlis with the Glis family (Bayesian posterior probability $(\mathrm{PP})=97$, Maximum Likelihood bootstrap value $(\mathrm{BS})=0.75$; Figure 2 ), and grouped NvNkl with the Nkl family ( $\mathrm{PP}=1, \mathrm{BS}=$ 0.99; Figure 2). Two of the three placozoan T. adhaerens candidate genes failed to group within the $\mathrm{gli} / \mathrm{glis} / \mathrm{nkl} / \mathrm{zic}$ superfamily (see Tad1.1.1692.1 and Tad1.40.97.1; Figure 2 ), but reciprocal BLAST searches suggested that they were related to ZF143 genes rather than members of the gli/glis/nkl/zic gene family. This is supported by phylogenetic analysis for Tad1.1.1692.1 ( $\mathrm{PP}=1, \mathrm{BS}=0.96$; Figure 2). Tad1.40.97.1 groups strongly with the ZF143 (PP = 1, $\mathrm{BS}=0.99$; Figure 2) genes rather than the $\mathrm{gli} / \mathrm{glis} / \mathrm{nkl} / \mathrm{zic}$ genes suggesting that it is also not a gli/glis/nkl/zic related gene. However the third $T$. adhaerens gene, Tadzic, grouped within the zic gene sub-family with strong support ( $\mathrm{PP}=1, \mathrm{BS}=0.99$; Figure 2 ). In the polychaete $C$. teleta, phylogenetic analyses strongly support the assignment of the three candidate genes, $C t$-zic, Ctgli and Ct-glis, into the zic, gli and glis sub-families, respectively $(\mathrm{PP}=1,1,0.97$ and $\mathrm{BS}=0.99,0.79$ and 0.75 , respectively; Figure 2). In addition, we cloned a partial sequence out of the acoel C. longifissura, Clozic, which grouped within the zic sub-family $(\mathrm{PP}=1, \mathrm{BS}=0.97$; Figure 2). The identification of Tadzic in Placozoa is the putative earliest metazoan zic ortholog identified to date. We conclude that zic genes were present in the common ancestor of Placozoa and later branching metazoans.
Two $\mathrm{gli} / \mathrm{glis} / \mathrm{nkl} /$ zic sequences were identified in $M$. leidyi despite extensive searches of the $M$. leidyi sequenced genome (50X sequence coverage, Ryan et al., manuscript in preparation). One $M$. leidyi sequence, Mlglis, grouped with the glis sub-family ( $\mathrm{PP}=0.97, \mathrm{BS}$ = 0.75; Figure 2). The second M. leidyi sequence, Mlgli, grouped with the gli sub-family $(\mathrm{PP}=1, \mathrm{BS}=0.79$; Figure 2). However, this grouping is based on the presence of only four of the five ZF domains, ZF2-ZF5 (Additional File 1). Attempts to identify the ZF1 domain via RACE PCR and sequence predictions were unsuccessful (data not shown). Thus, we cannot confirm that Mlgli contains all five ZFs. However, because it groups so strongly with the gli sub-family, we included it in our analysis. We confirmed the reported orthology of the three previously described $A$. queenslandica sequences. We identified an ancestral/sister gli/glis/nkl sequence (Amqgli2/3b, PP $=0.99, \mathrm{BS}=0.82$; Figure 2$)$, one glilike sequence (Amqgli2/3a, PP $=1, \mathrm{BS}=0.79)$, and one glis-like sequences (Amqglis1/3, PP $=0.95, \mathrm{BS}=0.65)$. Examination of the spacing between the cysteine residues in the ZF1 peptide sequence is also consistent with Mlglis, Amqgli2/3a, Amqgli2/3b and Amqglis $1 / 3$ sequences being more closely related to the $\mathrm{gli} / \mathrm{glis} / \mathrm{nkl}$ sub-families than the zic gene sub-family (Additional File 1). Degenerate PCR in a second sponge species (Ephydatia muelleri) failed to identify a poriferan zic sequence (data not shown). We conclude that no definitive zic ortholog exists in the ctenophore or poriferan animals analysed, but that both groups contain gli/glis/ $n k l$-like homologs in their genomes.

\section{Clozic and Ct-zic, but not Tadzic, contain ZF-NC domains}

As previous studies [1] have suggested that zic genes, but not gli or glis genes, contain a ZF-NC domain, we attempted to identify ZF-NC domains in the newly described zic genes Tadzic, Ct-zic and Clozic as well as in the $g l i / g l i s / n k l$ genes. We identified no ZF-NC or ZOC domains outside of the zic homologs. The ZF-NC domain is located immediately $\mathrm{N}$-terminal to the $\mathrm{ZF1}$ domain, so we aligned all the sequences $\mathrm{N}$-terminal to the ZF1 domains to identify candidate ZF-NC domains. We identified clear ZF-NC domains in CloZic, Ct-Zic and a partially conserved ZF-NC in TadZic (Figure 1B). The ZF-NC consensus sequence has been previously 
described as GAF(F/L)RYMRQP-(0-7AA)-IKQE [1]. $\mathrm{Ct}$-Zic and CloZic each have $87 \%$ and $93 \%$ conserved similarity with this consensus sequence across their respective ZF-NC domains (Figure 1B). However, the TadZic sequence is poorly conserved except for the presence of the four C-terminal amino acids, a conserved proline (Figure 1B), and a conservative arginine to histidine change at position 5 (Figure 1B). While CloZic and $\mathrm{Ct}$-Zic contain definitive ZF-NC domains, it is not clear if the TadZic sequence represents a functional ZF-NC domain.

\section{A single zic ortholog containing a ZOC domain was shared by Cnidaria and Bilateria}

Using non-biased alignment of the $\mathrm{N}$-terminal portions of all Zic proteins assayed, we identified a conserved RDFL-(1-2AA)-RR ZOC consensus sequence. The ZOC domain previously described consensus sequence was (S/T)RDFLxxxR [1,4]. However, our alignment suggests that the RDFL-(1-2AA)-RR consensus sequence is more prevalent throughout the metazoans than the previously described consensus. Using the novel consensus sequence, we identified a ZOC domain in NvZicA,C,D, E, Ct-Zic, and CloZic (Figure 1C). The NvZicA ZOC sequence is $100 \%$ identical to the new $Z O C$ consensus sequence. This is particularly important, because ZOC domains have not been previously described in cnidarians [1]. Our finding pushes back the emergence of the ZOC domain to at least the cnidarian-bilaterian common ancestor. The NvzicC-E genes possess more weakly conserved putative ZOC domains. However, in the case of NvzicD, the amino acid content of the domain HDYIHH, is $100 \%$ similar to the RDFL-RR sequence (Figure 1C), suggesting that the NvzicD ZOC domain may still be functional. Altering the definition of the ZOC domain results in the inclusion of a number of additional genes and taxa into the ZOC possessing zic group. They are the cnidarians (see NvzicA,C,D,E; Figure 1C), the vertebrate Zic4 and Zic5 sequences (see MmuZic5, XlaZic4; Figure 1C), nematodes (see CelRef2; Figure 1C), platyhelminthes (see DjaZicA; Figure 1C) and urochodates (see amphiZic; Figure 1C). We did not find a ZOC-like sequence for TadZic. However, due to sequence and annotation errors, it is possible that we have not identified a full length $\mathrm{N}$-terminal sequence and, thus, a ZOC-like domain may be encoded in the full Tadzic coding sequence. Using sequence similarity, it appears that putative ZOC domains can be identified in cnidarians, acoels, platyhelminthes, nematodes, annelids, vertebrates, insects and urochordates (Figure 1C). Thus, the earliest described ZOC sequence appears to have arisen in the cnidarian-bilaterian ancestor, rather than the urbilaterian ancestor.

\section{Nvzic expression during development of the cnidarian sea anemone $N$. vectensis}

We investigated the expression pattern of the five Nvzic genes during development of the cnidarian $N$. vectensis. NvzicA and NvzicB appear to be expressed at very low levels, as we were unable to obtain reproducible expression for both genes, although they are detected by RTPCR in 24, 48 and 72 hours post fertilization hpf cDNA samples suggesting that they are expressed at low levels beginning just after gastrulation and maintain expression into at least the planula larval stages (data not shown).

$N v z i c C-E$ genes all share similar expression patterns. $N v z i c C$ and $N v z i c D$ expression is first detectable in the presumptive tentacle ectoderm surrounding the mouth in planula larva (Figure 3B and 3G respectively). There are five notable differences between NvzicC and NvzicD expression patterns. First, $N v z i c D$ ectodermal expression is detected only in the presumptive tentacle domains (Figure 3G) and not in the intervening intertentacular ectodermal domains (compare Figure $3 \mathrm{G}$ and $3 \mathrm{H}$ with $3 \mathrm{~B}$ and $3 \mathrm{C}$ ), while $N v z i c \mathrm{C}$ is expressed in a ring encompassing both the presumptive tentacle and intertentacular domains (Figure $3 \mathrm{~B}, \mathrm{C}$ and $3 \mathrm{Q}$ ). In addition to $N v z i c C$ ectodermal expression, there might also be expression in the underlying endoderm. The second difference is that, by late planula stages, $N v z i c D$ expression is down regulated in the presumptive tentacle region (Figure $3 \mathrm{H}$ ), while $\mathrm{NvzicC}$ expression remains robust throughout planula and tentacle bud stages (Figures 3BD). Interestingly, $N v z i c D$ expression is up regulated again in the forming and early tentacles of the bud and juvenile polyp stages (Figure 3I and 3J). The third difference is that $N v z i c D$ expression in the juvenile polyp is detectable in distinct endodermal cells or groups of cells in the tentacles (Figure 3J and 3P), while NvzicC is detectable an ectodermal stripe in the proximal tentacle region (Figure 3E). It is currently unclear which cell types are expressing $N v z i c D$. The fourth difference is that $N v z i c D$ can be observed in individual cells in the polyp body wall ectoderm (Figure 3G and 3S; arrowheads). Lastly, $N v z i c D$ expression is detectable in distinct cell populations in the endodermal component of the developing mesenteries (Figure 3T; closed arrow) and endoderm surrounding the pharyngeal ectoderm (Figure 3T; open arrow), while $N v z i c C$ is not.

$N v z i c E$ expression is first detected during embryonic stages, which is earlier than either $N v z i c C$ or $N v z i c D$. It is weakly expressed throughout the embryonic ectoderm shortly after gastrulation (Figure $3 \mathrm{~K}$ ). At planula stages, weak ubiquitous expression of NvzicE is detected throughout the ectoderm but enrichment is observed in oral ectoderm and presumptive tentacle bud domain and at the aboral pole in the apical tuft (Figure 3L; arrow and 
arrowhead, respectively). By late planula stages $N v z i c E$ expression is down regulated in the body wall ectoderm but remains detectable in the apical tuft ectoderm (Figure $3 \mathrm{M}$; arrow) and the presumptive tentacle domain (Figure $3 \mathrm{M})$. By the tentacle bud stage, aboral expression of $N v z i c E$ is no longer detectable (Figure $3 \mathrm{~N}$ ), due to the loss of the apical tuft after settlement. However, expression persists in tentacle endoderm (Figure 3O) and, occasionally, single NvzicE-positive cells appear in the tentacle ectoderm in juvenile polyps (Figure 3R). In summary, NvzicC-E display similar expression patterns in the developing tentacle endoderm and ectoderm, the endodermal component of the mesenteries and in the oral ectoderm. In addition, $N v z i c E$ is expressed in the apical tuft ectoderm

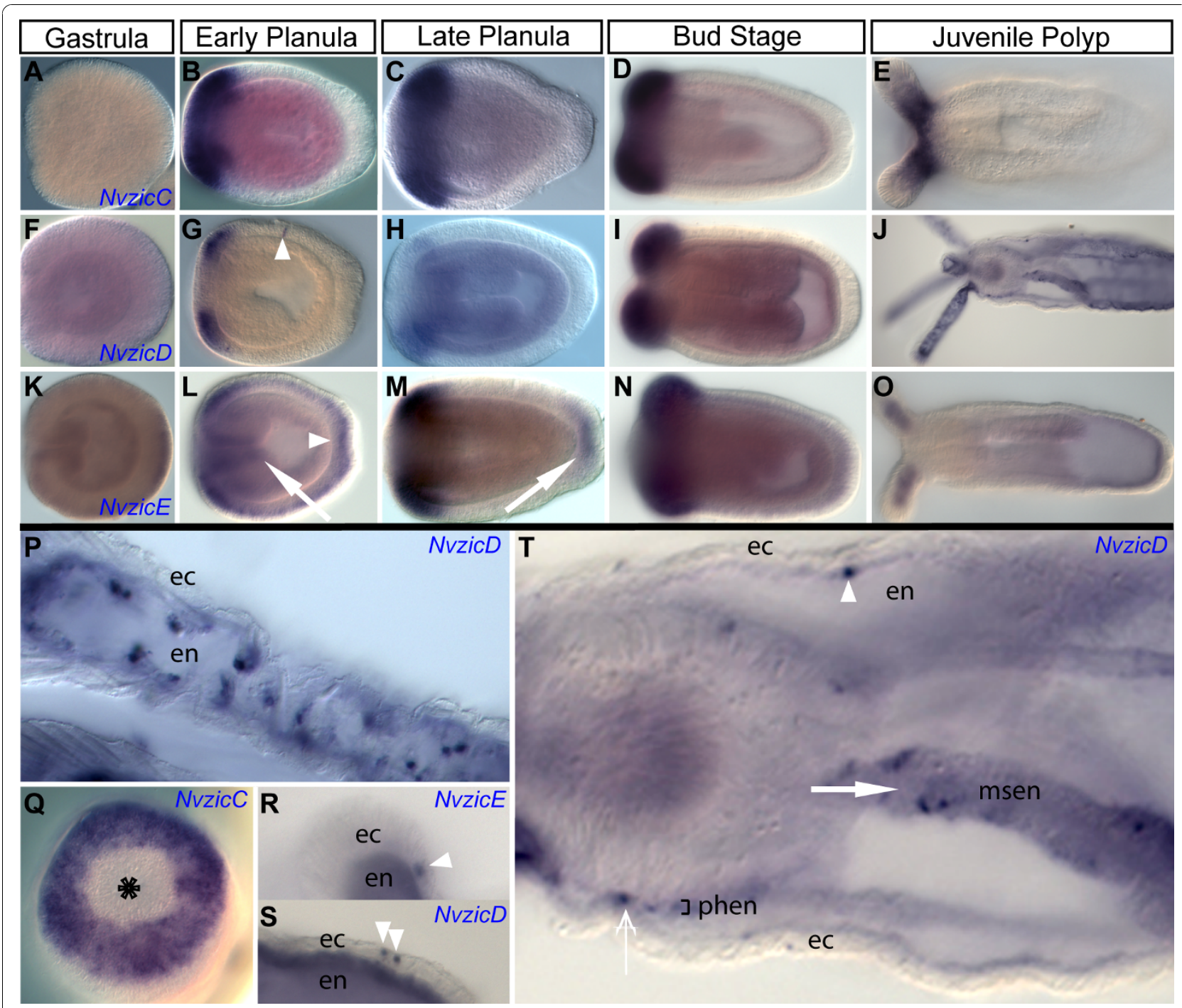

Figure 3 Nvzic expression in Nematostella vectensis. Expression of Nematostella NvzicC (A-E,Q), NvzicD (F-J, P, S, T) and NvzicE (K-O, R). Late gastrula $(A, F, K)$, early $(B, G, L)$ and late $(C, H, M, Q)$ planula, bud $(D, I, N)$ and polyp $(E, J, O, R, S, T)$ stages are shown. All images are lateral views with oral to the left except $\mathrm{Q}$, which is an oral view with mouth indicated by an asterisk. The endoderm is indicated by en, ectoderm by ec, pharyngeal endoderm by phen and mesentery endoderm by msen. NvzicC is expressed in presumptive tentacle and tentacle bud ectoderm (B, C and D) but not in oral ectoderm (Q) in planula stages. The polyp NvzicC expression is in the tentacle ectoderm proximal to the polyp body, but not in distal regions (E). NvzicD is expressed in the presumptive tentacle ectoderm ( $\mathrm{G}$ and $\mathrm{H}$ ) and individual ectodermal cells ( $\mathrm{G}$, arrowhead) of the planula. NvzicD is also expressed in tentacle buds (I), tentacular endoderm ( $\mathrm{J}$ and $\mathrm{P})$, pharangeal endoderm $(\mathrm{T}$, open arrow) and in distinct cells in the endodermal component of the directive mesentaries ( $T$, closed arrow) in the polyps. The polyp NvzicD expression is occasionally observed in the endodermal cells ( $\mathrm{T}$. arrowhead) and the ectodermal cells ( $\mathrm{S}$, arrowheads) of the polyp. NvzicE expression is expressed in the aboral pole of the gastrula embryo ( $K$, arrow). NvzicE is expressed $n$ the oral ectoderm ( $L$, arrow), presumptive tentacle $(L$ and $M)$ and the apical tuft $(\mathrm{M}$, arrow) the planula and tentacle buds $(\mathrm{N})$, tentacle endoderm $(\mathrm{O})$ and occasional individual ectodermal cells in the tentacles $(\mathrm{R}$, arrowhead). 
of the late embryonic stages and in individual cells throughout the body of the developing animal.

\section{Ct-zic expression during development of the bilaterian lophotrochozoan C. teleta}

Prior to this study zic gene expression had only been investigated in a single lophotrochozoan, the oligochaete T. tubifex. Most protostome zic expression has been described in the ecdysozoans. Thus, investigating additional lophotrochozoan animals will provide a more complete comparison for understanding the relationship among bilaterian $z i c$ gene expression patterns.

$C t$-zic expression in C. teleta is first detected during cleavage stages in a subset of micromeres at the animal hemisphere of the blastula (Figure 4M). Ct-zic is not detectable in endodermal vegetal macromeres at any stage examined. At later stages, during tissue morphogenesis (stages 3 - 9), Ct-zic transcript is detected in the neural ectoderm and mesodermal cell types. Neural ectoderm expression begins at stage 3 in bilaterally symmetric subsets of the anterior neural ectoderm (Figure $4 \mathrm{~B}$; arrow), which is also visible from an anterior view at stage 4 (Figure $4 \mathrm{~N}$ ). Ct-zic expression is maintained in this anterior neural ectodermal domain throughout development and is present in a sub-region of the developing brain which is clearly visible by stage 6 (Figure $4 \mathrm{H}$; arrow). Previous fate mapping in C. teleta has demonstrated that the majority of brain neurons are derived from the anterior neural ectoderm $[21,22]$ and it is likely that $C t$-zic neurons arise from $C t$-zic positive domains in the overlying ectoderm.

Neural ectodermal expression of $\mathrm{Ct}$-zic is also detected along the lateral edge of the ventral neural ectoderm at stage 4 (Figure $4 \mathrm{C}$ and $4 \mathrm{O}$, arrows). The lateral ectodermal $C t$-zic domain expands toward the posterior of the trunk (Figure 4F), and is then down-regulated in an anterior to posterior wave, visible by late stage 6 (Figure 4G; white lines mark anterior border of expression). From stages $7-9, C t$-zic expression in the lateral ectoderm is maintained in ventral-lateral cells in the growth zone (Figure 4I, arrow), where new segments are forming. Although the exact lateral border of the ventral neural ectoderm, which forms the ventral nerve chord (VNC), has not been mapped, we think that lateral ectodermal Ct-zic expression is within the ventral neural ectoderm. In support of this hypothesis, early neurogenic markers (Ct-ash and Ct-neurogenin; unpublished observations) positionally overlap with $\mathrm{Ct}$-zic expression, although expression of the neurogenic markers extends more medially than $C t$-zic at similar stages. Furthermore, we observe $C t$-zic in the VNC beginning at stage 6 (Figure 4G, arrowhead). Ct-zic positive cells in the $\mathrm{VNC}$ are first detected in anterior segments where $C t$ $z i c$ expression is no longer apparent in the ventral- lateral ectoderm (Figure 4G). By stage 8, Ct-zic positive cells are positioned medially and laterally within the VNC (Figures $4 \mathrm{~K}$ and $4 \mathrm{Q}$ ). Thus, we conclude that $C t$ $z i c$ is expressed at the lateral edge of the presumptive ventral neural ectoderm, in the VNC, and in a ventrallateral ectodermal domain in the growth zone where new segments are forming.

Mesodermal expression of $\mathrm{Ct}$-zic is detectable at stages 3 to 9 . Beginning at stage 3, expression is in one or a few cells in an anterior subset of the bilateral mesodermal bands (Figure 4A and 4B). At stages 4 and 5, mesodermal expression is detected in domains lateral to the foregut (Figure $4 \mathrm{C}$ and $4 \mathrm{E}$, arrowheads). The expression around the foregut expands during stages $6-7$ (Figure 4G and 4I) and is in a sub-set of the Ct-twist positive visceral mesoderm surrounding the foregut [23] (Figure 4I, notched arrowhead). In addition, Ct-zic expression is observed within a subset of Ct-twist-positive anterior mesoderm in the head (Figure 4I, arrowhead).

There is additional $C t$-zic mesodermal expression in four longitudinal stripes (two on each side of the larva) of segmentally-reiterated cell clusters. This expression domain is first visible in a few cells at stage 5 (Figure $4 \mathrm{E}, 4 \mathrm{~F}$ and $4 \mathrm{O}$, arrowheads). The number of $\mathrm{Ct}$-zic-positive cell clusters increases along the anterior-posterior (A-P) axis as the animal elongates and new segments form (Figure 4J and 4L, arrowheads). The A-P expansion of this pattern is reminiscent of the expansion of Ttu-zic expression in chaetal sac associated mesoderm [11]. By stages 7 and 8, when chaetae are present, Ct-zic expression can be clearly observed in cells wrapping the chaetal sacs (Figure 4J, 4L and 4P) but not in the chaetal sacs themselves (Figure 4P, arrows), indicating that the zic-positive cells are chaetal-sac associated mesoderm. $C t$-zic expression is maintained in these structures through stage 9. In summary, mesodermal expression of $C t$-zic is found in the anterior portion of mesoderm bands at stage 3 , in the visceral mesoderm surrounding the foregut, in the head mesoderm at the anterior tip of the animal and in mesoderm associated with forming chaetae.

\section{Mlglis and Mlgli expression during development of the ctenophore M. leidyi}

No definitive zic homolog was identified in either Porifera or Ctenophora but phylogenetic analyses identified gli/glis/nkl-like sequences in both clades (Figure 2). We investigated the expression pattern of Mlglis and Mlgli during the development of the ctenophore comb jelly M. leidyi to gain insight into the evolution of gli/glis/ $n k l / z i c$ expression patterns as the family expanded during metazoan evolution. Both genes showed expression in putative sensory structures in the floor of the apical 
organ. However, Mlgli expression was not detected until very late cydippid larval stages (Figure 5I and 5J) and expression was weaker than the robust expression observed for Mlglis.

Mlglis expression during M. leidyi development is exclusively associated with the apical organ and is not detected in any other tissues. Expression is first detected upon the completion of gastrulation in two opposing groups of cells in the presumptive apical organ ectoderm at the aboral pole of the developing embryo (Figure $5 \mathrm{~A}$ ). Over the next hour of development, expression expands into two arches around the floor plate ectoderm of the apical organ (Figure 5B). The number of cells in each domain expands from approximately four to twenty, though it is not clear if the expansion is due to nascent expression of Mlglis in adjacent cells or via cell proliferation of the Mlglis expressing cells. Over the next three hours, Mlglis domains assume a dumbbell shape and eventually bifurcate (Figures $5 \mathrm{C}-\mathrm{E}$ ), resulting in four distinct domains of expression in the floor plate of the apical organ (Figure 5F). The apical organ contains a statocyst consisting of four groups of cells that give rise to balancing cilia that support the mineral containing lithocytes. The statocyst functions as a balance

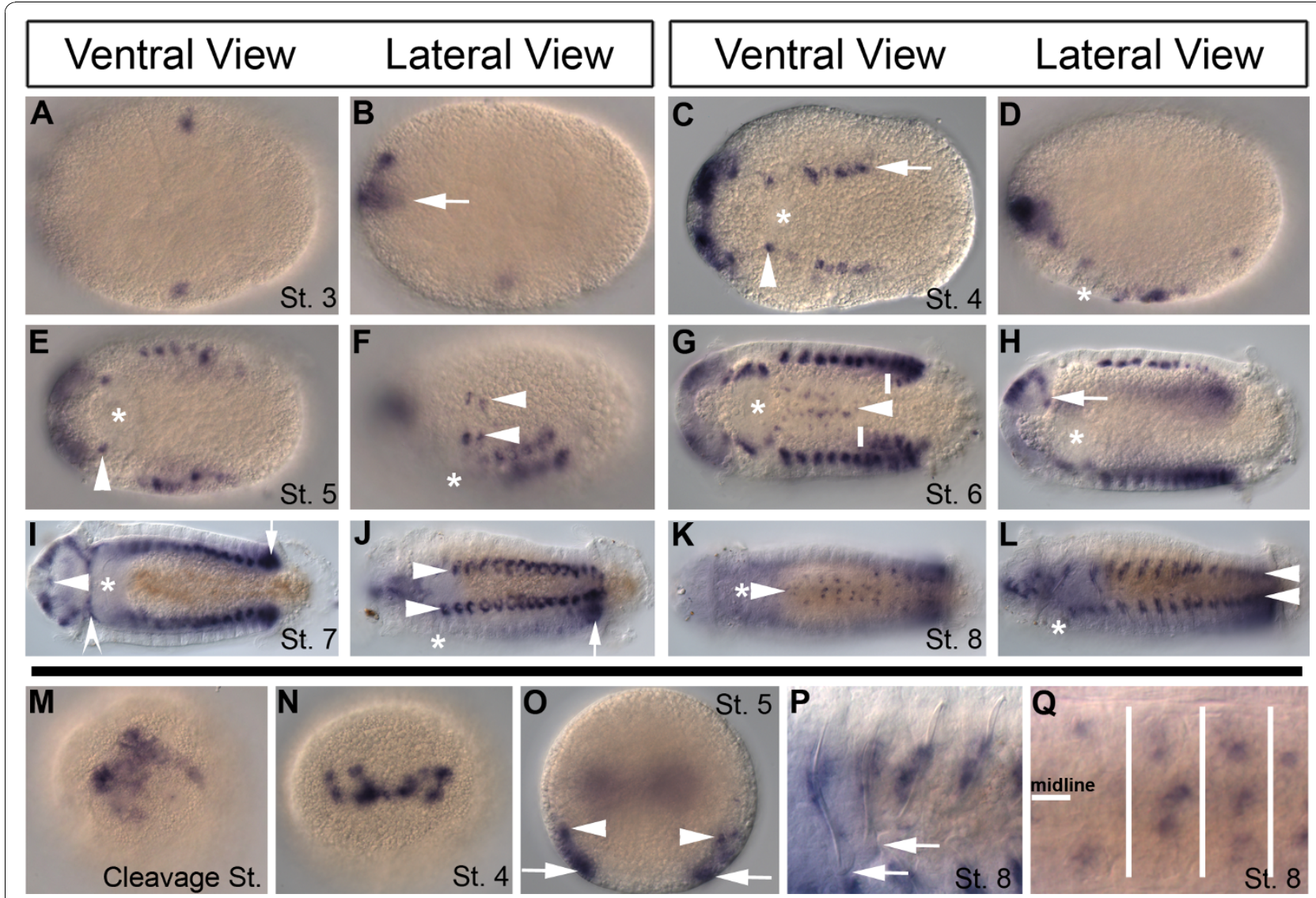

Figure 4 Capitilla teleta-zic (Ct-zic) expression in the lophotrochozoan C. teleta. Expression of Ct-zic at cleavage (M), stage 3 (A and B), stage $4(C, D$ and $N)$, stage $5(E, F$ and $O)$, stage $6(G$ and $H)$, stage $7(I$ and, J) and stage $8(K, L, P$ and $Q)$. Views are as indicated except that $H$ is a ventral-lateral view; $\mathrm{M}$ is an animal view; $\mathrm{N}$ and $\mathrm{O}$ are anterior views; $\mathrm{P}$ is a lateral view; and $\mathrm{Q}$ is a ventral view. In all lateral and ventral views the anterior is to the left and the ventral is down in all lateral views. An asterisk $\left(^{*}\right)$ indicates the relative mouth position. Ct-zic is detectable in micromeres on animal pole (M). Ct-zic mesodermal expression is detected in the anterior mesodermal band on the ventral side of stage 3 animals ( $A$ and $B$ ) and in two bilateral mesoderm domains adjacent to the foregut at stages 4 and 5 ( $C$ and $E$, arrowheads). The mesodermal expression expands around the foregut during stages $6(\mathrm{G})$ and 7 (I, notched arrowhead). At stage 5, four lateral mesodermal domains ( $F$, arrowheads) are detected. At stage 7, lateral mesoderm domains form two longitudinal rows along the anterior-posterior (A-P) axis $(\mathrm{J}$, arrowheads). At stage 8 , the longitudinal domains are clearly mesoderm associated with chaetae ( $L$, arrowheads, and $\mathrm{P})$, but not the chaetal $\operatorname{sac}(\mathrm{P}$, arrows). Ct-zic is expressed in presumptive brain ectoderm in all stages ( $\mathrm{B}$, arrow, $C-L$ and $N)$. Ventrolateral expression at the lateral edge of ventral neural ectoderm is detected at stage $4(\mathrm{C}$, arrow) and stage 5 ( $\mathrm{F}$ and $\mathrm{O}$, arrows). The lateral neural ectoderm domain is downregulated in an A-P wave apparent at stage 6 (G, white line demarcates anterior border of expression) but remains expressed in the growth zone (I and J, arrows). Ct-zic is also detectable in neurons in the brain ( $\mathrm{H}$, arrow) and, beginning at stage 6 , in the ventral nerve cord (VNC; $\mathrm{G}$, white arrowhead). VNC expression persists through stage 8 (K, arrowhead, and $\mathrm{Q})$. Medial and lateral ganglions are detected in each segment $(\mathrm{Q}$, which is a higher magnification of $\mathrm{L}$ ). In $\mathrm{Q}$, the white lines demarcate segmental boundaries. 


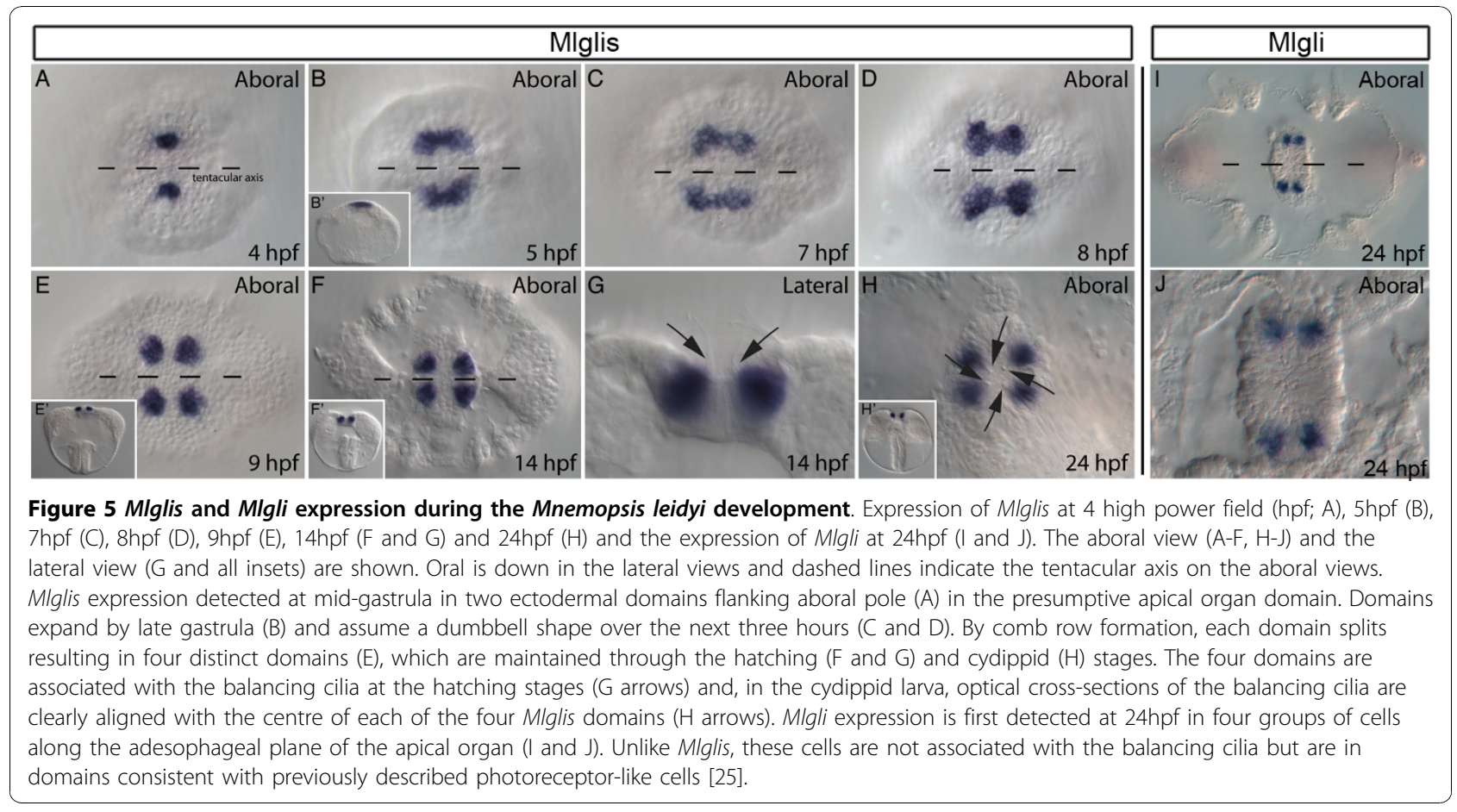

organ allowing recognition of proper body orientation within the water column. This is achieved by sensing the gravitational pull on the lithocytes perched on the four groups of balancing cilia [24]. Mlglis expression is detected in the statocyst cells associated with the balancing cilia (Figure 5G and 5H, arrows) which, based on function, are putative neuronal or neuronal-like cells. In conclusion, Mlglis is expressed in the presumptive

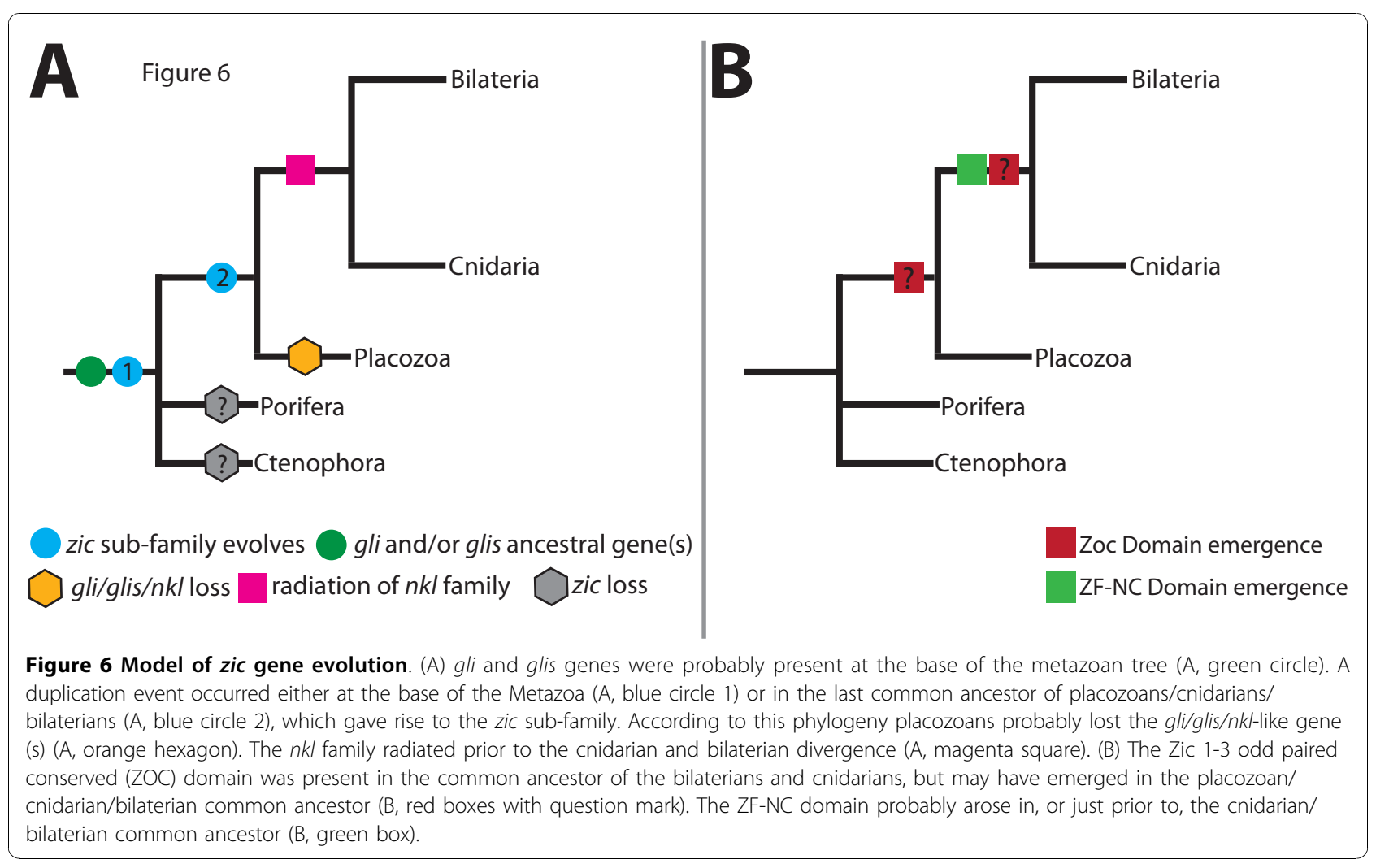


balancing cilia of the apical organ statocyst beginning shortly after gastrulation and expression is maintained throughout development.

Mlgli expression is first detected in 24-hour-old larvae in four groups of putative sensory cells in the floor of the apical organ. Mlgli-expressing cells are symmetrically arranged within the floor of the apical organ in the $24 \mathrm{hpf}$ cydippid larva (Figure 5I and 5J). Based on their position, the Mlgli-expressing cells and the Mlglis expressing cells represent two distinct populations of cells (compare Figure 5J with 5H). The Mlgli expressing cells are not associated with the balancing cilia and are positioned further from the centre along the adesophageal plane of the apical organ than the Mlglis expressing balancing cilia cells. The domains exhibiting Mlgli expression are also associated with putative sensory structures. Mlgli expression in the apical organ is reminiscent of previously identified structures, which morphologically resemble photoreceptors by electron microscopy [25]. We conclude that Mlgli is expressed in the putative photoreceptive cells in the apical organ and has a distinct pattern from that observed for Mlglis.

\section{Discussion}

\section{The origin of the metazoan zic gene}

Understanding the origin of the zic gene family is ultimately tied to the understanding of the branching pattern for basal metazoans. There are four basally branching metazoan clades - the cnidarians, placozoans, ctenophores and poriferans. Previous work and our data demonstrate that $z i c$ genes were present in the placozoan and cnidarian clades, but absent from the current poriferan and ctenophore data sets (Figure 1). Although the phylogenetic relationship between basal metazoans currently is debatable [26-30], the prevailing hypotheses place cnidarians as the sister group to the bilaterians and the divergence of placozoans after the origin of both poriferans and ctenophores [26-29]. Thus, the simplest model is that zic family genes diverged once in the common ancestor of the placozoans/cnidarians/bilaterians (Figure 6A, blue circle 2). The alternative hypothesis that zic genes evolved earlier (Figure 6A, blue circle 1) but were lost in more basally branching clades (Figure $6 \mathrm{~A}$, grey hexagons) and maintained in placozoans, cnidarians and bilaterians - cannot be ruled out. However, given the conserved expression of zic genes in mesodermal and neural tissues, the notion that zic genes would be maintained in the placozoans, which have no definitive mesoderm or neural cells, but lost in an ctenophores, which possess muscle cells and a clear nervous system, seems less likely than the notion that zic genes evolved prior to placozoan divergence, but after the ctenophore and poriferan radiations. The $T$. adhaerens genome does not possess clear gli/glis/nkl-like homologs [31], which may have been lost in conjunction with the loss of these tissues in the placozoan lineage. The simplest model is a single zic divergence event in the last common ancestor of the placozoans, cnidarians and bilaterians.

Regardless of when zic genes evolved, they probably diverged from a $g l i / g l i s / n k l$-like gene. The basally branching ctenophore and poriferans likely possessed at least two gli/glis-like genes, arguing that a gli/glis/nkllike gene, which is lacking in non-metazoans, was present and already duplicated at least once at, or near the time of, the emergence of the earliest metazoans. The presence of the poriferan Amqgli2/3b sequence suggests that a third $g l i / g l i s / n k l$-like gene may have existed prior to the divergence of poriferans and ctenophores. However, Amqgli2/3b may also represent a lineage specific duplication. As the $g l i / g l i s / n k l$ clades represent a single sister group to the zic clade, it is unclear whether a $g l i$, glis or $n k l$ gene duplication/divergence would have given rise to the zic family. No clear $n k l$ genes are present in the basal metazoans, except for the cnidarians, arguing that duplication of a gli or glis-like gene resulted in the divergence of the zic family. However, the existence and phylogenetic position of the Amqgli2/3b gene raises concerns about this model. Duplication or divergence of an Amqgli2/3b-like gene could have also given rise to the zic family. Further resolution of the branching pattern of basal metazoans and sampling for $\mathrm{gli} / \mathrm{glis} / \mathrm{nkl} / z i c$ genes in ctenophore and poriferan species is required before more a finite hypotheses can be drawn about the evolutionary origin and precursor of the zic gene family.

\section{Two distinct $g$ li similar (glis) sub-families exist}

Though the glis and $n k l$ gene sub-family names have been used interchangeably, $n k l$ should uniquely identify the glis2/sug gene family. glis2 genes were originally described as neurogenic kruppel like $(n k l)$ genes [32], while the glis1/3 genes had been identified as gli similar [33]. Our data suggest that the $n k l$ and glis genes represent two distinct clades. The glis clade consists of glis1, glis3 and the D. melanogaster lameduck ( $\operatorname{lm} d)$ homologs (Figure 2), and the $n k l$ clade consists of glis2 and D. melanogaster sugarbabe (sug) homologs (Figure 2). This distinction is also supported by a recent investigation of $\mathrm{C} 2 \mathrm{H} 2 \mathrm{ZF}$ genes in amphioxus. The authors of that study identified two distinct strongly supported glis clades for glis 2 and glis1/3 genes [34]. We propose that the $n k l$ gene sub-family name should be used to uniquely identify the sug and glis2 genes as a distinct gene sub-family.

\section{ZOC and ZF-NC domains pre-date emergence of Bilateria}

The cnidarian-bilaterian zic ortholog contained a ZFNC-like domain. The first eight amino acids of the published ZF-NC conserved sequence (GAFFRYMR(0-7aa) IKQE) [1] are $86 \%$ identical and $100 \%$ similar in the 
putative ZF-NC domain of NvZicA (GAFFRFMR; Figure 1B). In most ZF-NC sequences characterized to date, the arginine residue at position 8 is followed by a QPIKQE sequence (Figure 1B). The corresponding NvZicA sequence (SPAKDN) is $50 \%$ similar to the Cterminal portion of the ZF-NC domain (Figure 1B). Lastly, although the first part of the consensus domain is missing in the TadZic sequence, the last four amino acids (AKQE) are $75 \%$ identical and $100 \%$ similar to the consensus sequence. As no functional mapping has been conducted on the ZF-NC domain and there are strong sequence similarities between the ZF-NC regions of early branching metazoans and bilaterians, it seems likely that presence of a ZF-NC domain may predate the urbilaterian zic gene (Figure 6C, green square).

NvZicA-E each contain all, or some of, the reported, and/or proposed, ZOC consensus sequence or have amino acids in similar functional groups to the consensus sequence (Figure 1C). In the case of NvZicE, the actual sequence is not identical to the consensus sequence, but $100 \%$ similarity is observed in the five Nterminal amino acids (Figure 1C). In fact, the NvZicE sequence is arguably more similar to the consensus sequence than the oligochaete Ttu-Zic sequence (compare NvZicE and TtuZic; Figure 1C), which has a ZOC domain [1]. Thus, we suggest that the conserved ZOC and ZOC-like domains in the Nematostella Nvzic genes supports the inclusion of the ZOC domain in the cnidarian-bilaterian ancestral zic gene (Figure 6C, red square). Our results suggest that previously undescribed ZOC domains are present in platyhelminthes, nematode and urochordate zic genes. Thus, all sampled bilaterian clades to date now have at least one lineage with a zic gene containing a $\mathrm{ZOC}$ domain.

ZOC domains have been shown to have two functions in in vitro transcription assays. The MmuZic2 ZOC domain has been demonstrated to increase Zic transcription factor activity and to bind the transcription factor Mfa-1 [4]. Currently it is not clear if the ZOC domain increases Zic transcription factor activity via intramolecular interactions, intermolecular interactions or both in vivo. Future studies focused on characterizing the activity of cnidarian $\mathrm{ZOC}$ sequences are required in order to verify the existence of functional ZOC domains in the cnidarians. Identification and comparative analysis of ZOC interacting proteins in both cnidarian and bilaterian lineages may be useful for determining the molecular relationships of zic gene function in metazoan development and evolution.

\section{Evolution of mesodermal zic expression}

Mesodermal expression of zic genes is present throughout the Bilateria. In D. melanogaster, opa is expressed broadly throughout the mesoderm primordia of the segmented region of the embryo [8]. In both lophotrochozoans studied thus far (T. tubifex and C. teleta) zic is expressed in a segmentally reiterated pattern in mesoderm associated with the chaetal sacs [12] (Figure 4) and, in chordates, zic expression is observed in a segmented pattern in dorsal domains of forming somites. In addition, within protostomes, zic homologs are expressed in visceral mesoderm [surrounding foregut in C. teleta (Figure 4) and around a portion of the forming midgut in D. melanogaster] [8]. In Cnidaria, we observe Nvzic expression in the gastrodermis, a bifunctional endomesodermal tissue, in the forming tentacles, around the pharynx and in the endomesodermal component of the directive mesenteries (Figure 4).

One hypothesis raised by our data is that bilaterian mesodermal zic expression is derived, at least in part, from the endomesodermal expression that existed in the common ancestor of the Bilateria and Cnidaria. The expression of NvzicD and NvzicE in tentacle endomesoderm and NvzicD in endomesoderm surrounding the pharynx supports this hypothesis. The tentacles are capable of multiple complex movements, including capture and transport of food to the oral opening due to the high density of myoepithelial cells. It is possible that there are similarities between tentacle endomesoderm and bilaterian musculature. Future work characterizing cell types expressing NvzicD and NvzicE and comparing the zic function in $N$. vectensis endomesoderm will provide an insight to the overall relationship of cnidarian endomesoderm to bilaterian endoderm and mesoderm.

\section{zic expression in neural development}

Our data support an ancestral role for zic genes as regulators of neural sub-type development. Expression and function of zic homologs in neural development is present throughout the Bilateria. However, only in the chordate lineage does zic expression and function include the entire neurogenic region in the early embryo. This suggests that, though zic genes have a conserved neural role in bilaterian animals, the ancestral role was probably sub-type specification. Nvzic genes are expressed in the presumptive and developing tentacle buds in both the endoderm and ectoderm in the cnidarian $N$. vectensis (Figure $3 \mathrm{~J}$ and $3 \mathrm{O}$ and $4 \mathrm{E}$, respectively). The tentacles are highly neuralized structures [35], suggesting that zic may be regulating tentacular neurogenesis. In support of this hypothesis, a number of neural genes (Nvelav, NvanthoRF, Nvmushashi, Nvgcm) and cross-reactive antibodies against the serotonin and GABA neuropeptides are detected at high levels in the developing tentacle endoderm and ectoderm [35]. In addition, NvzicE expression in the forming apical tuft sensory organ (Figure $3 \mathrm{M}$ ) is consistent with a role in neural development. zic expression in the apical tuft of 
echinoderms has also been described [36]. We describe expression of Mlglis and Mlgli in the ctenophore M. lei$d y i$ in neural structures of the developing apical organ (Figure 5). Thus, we suggest that the role of zic genes in neural development may have been shared with a gli/ glis/nkl/zic ancestral gene. This is also supported by the observations that gli, glis and $n k l$ genes all have described roles in metazoan neurogenesis [2,3]. Previous studies and our data, taken together, support the hypothesis that one of the ancestral roles of the zic gene family was to specify neural sub-types.

\section{Zic homologs may have been co-opted to pattern segmentally reiterated structures along the A-P axis in Bilateria}

In the lophotrochozoans T. tubifex and C. teleta, zic is expressed in mesoderm associated with segmentally reiterated chaetae. In amphioxus and vertebrates, zic homologs are expressed in the dorsal region of somites. Both somitic expression and mesodermal expression associated with chaetae indicate a role in segmental musculature formation for zic homologs in lophotrochozoans and chordates. C. teleta zic homologs are also expressed in a reiterated pattern in the ventral nerve cord, reflecting the segmental organization of its body plan (Figure 4). Likewise, opa has a role promoting the proneural gene achaete expression in each segment during D. melanogaster neurogenesis [9]. opa mutants were originally identified for their pair-rule phenotype (every other segment disrupted) [37]. However, opa's ubiquitous expression throughout the presumptive segmented region is distinct from the typical seven-stripe pair-rule pattern. In addition, opa is required for proper expression of the $w g$ (wnt) segment polarity gene in all parasegments [7]. Together, these data suggest that zic homologs function to pattern segmentally reiterated structures and molecular domains in bilaterians, but are unlikely to function directly in the segmentation process. Rather, the lack of similarity between zic expressing reiterated structures across bilaterian lineages suggests that $z i c$ genes, due to their ancestral roles in neural and mesoderm development in a cnidarian-like ancestor, have been co-opted multiple times downstream of the segmentation programme to pattern various segmentally iterated structures.

\section{Conclusions}

Based on our analysis, we propose that the first metazoan zic arose from a gli/glis/nkl-like gene prior to the emergence of the Placozoa, but after the divergence of both Ctenophora and Porifera. ZOC and ZF-NC domains were probably present in the ancestral zic gene shared by the cnidarian-bilaterian common ancestor. We hypothesize that the metazoan zic neural expression, which is present in all animals assayed thus far, may be derived from the expression of an ancestral gene in sensory cells. We also propose that the mesodermal expression of bilaterian zic genes may be derived from gastrodermal expression of zic homologs in a cnidarianbilaterian ancestor. Lastly, we suggest that zic genes have been co-opted multiple times down stream of segmentation programmes to pattern segmentally reiterated structures in some bilaterian clades. In summary, our results raise an interesting hypothesis about the molecular link between cnidarian endomesoderm and bilaterian mesoderm, and suggest that a role for zic genes in neurogenesis may be an ancient metazoan feature.

\section{Methods}

\section{Identification of candidate gli/glis/nkl/zic genes}

In order to identify candidate zic genes we searched published genomic resources, Trichoplax adhaerens http://genome.jgi-psf.org/Triad1/Triad1.home.html, Nematostella vectensis http://genome.jgi-psf.org/ Nemve1/Nemve1.home.html, Capitella teleta http://genome.jgi-psf.org/Capca1/Capca1.home.html, the unpublished genome of the ctenophore Mnemiopsis leidyi (NIH), the Amphimedon queenslandica http://spongezome.metazome.net/cgi-bin/gbrowse/amphimedon/ and using keyword or TBLASTN and BLASTX searches http://www.ncbi.nlm.nih.gov/BLAST/. We also used previously identified degenerate PCR primers [1] in order to attempt to clone zic fragments from the mixed stage embryonic cDNA acoel Convolutriloba longifissura, sponge Ephydatia muelleri and ctenophore Mnemiopsis leidyi using previously described zic specific primers [1]. Degenerate fragments were cloned by TA cloning into the pGEM-T Easy vector (Promega, CA, USA) and sequenced (Macrogen, Seoul, Korea). Sequences were used to design nested 5' and 3' RACE primers for each candidate sequence. Full-length sequences were obtained by compiling $5^{\prime}$ and 3' RACE sequences. Finally, sequences were confirmed by amplifying the full length sequence from mixed stage embryonic cDNA.

\section{Phylogenetic analysis}

Full length sequences for Gli, Glis and Zic proteins were aligned using the Muscle Alignment [38] web server http://www.ebi.ac.uk/Tools/muscle/index.html. The protein sequences were trimmed using the $\mathrm{N}$-terminal cysteine residue of the ZF1 domain and C-terminal histidine residue of the ZF5 domain to demarcate the five $\mathrm{C} 2 \mathrm{H} 2 \mathrm{ZF}$ domains (Additional File 1). A Bayesian phylogenetic analysis was carried out using MrBayes 3.1.2 [19] with a mixed protein model for 1,000,000 generations sampled every 100 generations with four chains. The Jones model was chosen with a posterior probability of 1 . A summary 'consensus' tree was generated in 
MrBayes using the last 7500 trees (30,000 total trees) representing 750,000 stationary generations. Posterior probabilities were calculated from the 'consensus' tree. A maximum likelihood analysis (using PhyML 3.0 [20]) was also conducted. The Jones model is not present in PhyML 3.0 so the JTT model of evolution (selected via ProtTest [39]) was used with 1000 bootstrap replicates to find the most likely tree: the JTT model is not present in MrBayes. Thus, identical models could not be used for each analysis.

\section{Alignment of ZOC and ZF-NC domains}

The C-terminal domain of full-length alignments derived from Muscle alignment (above) was trimmed from the third amino acid after the first cysteine residue of ZF1. The remaining N-terminal portions of the proteins were then realigned using Muscle and viewed using MacVector version 11.1.1 (MacVector, Inc, NC, USA).

\section{RNA in situ hybridization and image analysis}

We performed in situ hybridizations, as previously described, for Mnemiopsis leidyi [40], N. vectensis [32] and C. teleta [21]. All images for M. leidyi and N. vectensis were acquired using a Zeiss Axioskop 2 in conjunction with the Axiocam HRc and Axio vision 4.7 software (Zeiss), Jena, Germany. C. teleta images were acquired using a Zeiss Axioskop 2 microscope and a SPOT Flex digital camera (Diagnostic Instruments, Inc, MI, USA) in conjunction with the Spot Advanced version 4.6 software (Diagnostic Instruments, Inc). Additional image processing was done with Helicon Focus software (Helicon Soft Ltd. Kharkov, Ukraine) for some images as indicated in figure legends. Additional images for $N$. vectensis and M. leidyi genes shown here are available in the Kahi Kai image database http://www. kahikai.org/.

\section{Additional material}

Additional file 1: Alignment of Gli, Glis, Nkl and Zic protein znc

finger (ZF) domains. An alignment of the five tandem $\mathrm{C}_{2} \mathrm{H} 2 \mathrm{ZF}$

domains that define the Gli/Glis/Nkl/Zic family is shown. The alignment

begins with the first cysteine of ZF1 and ends with last histidine of ZF5.

\begin{abstract}
Abbreviations
AP: anterior-posterior; BS: bootstrap; Ct-zic : Capitella teleta zic homolog; hpf: hours post fertilization; PCR: polymerase chain reaction; PP: posterior possibility; VNC: ventral nerve cord; ZF: zinc finger; ZOC: Zic 1-3 oddpaired conserved.
\end{abstract}

\section{Acknowledgements}

We would like to thank Sally Leys and Pamela Windsor for providing Ephydatia muelleri cDNA and RACE cDNA, and Andreas Hejnol for providing Convolutriloba longifissura RACE CDNA and help in obtaining Convolutriloba longifissura embryonic material. We would also like to thank Joseph F Ryan, Andreas D Baxevanis and the NIH/NISC Comparative Sequencing Program for allowing access to the draft sequences of the ctenophore Mnemiopsis leidyi genome. This work was made possible by funding from The National Institutes of Health, Eunice Kennedy Shriver National Institute of Child Health and Human Development F32HD055000 and the National Center for Research Resources G12RR003061.

\section{Authors' contributions}

MJL conceived the study, performed the phylogenetic analysis, performed the $N$. vectensis expression analysis and cloned the zic homologs. NPM performed the C. teleta expression analysis. KP performed the M. leidyi expression analysis and the blast searches of the unpublished M. leidyi genome. All authors participated in the drafting and all approved the manuscript.

\section{Competing interests}

The authors declare that they have no competing interests.

Received: 31 May 2010 Accepted: 5 November 2010

Published: 5 November 2010

\section{References}

1. Aruga J, Kamiya A, Takahashi H, Fujimi TJ, Shimizu Y, Ohkawa K, Yazawa S, Umesono Y, Noguchi H, Shimizu T, Saitou N, Mikoshiba K, Sakaki Y, Agata K, Toyoda A: A wide-range phylogenetic analysis of Zic proteins: implications for correlations between protein structure conservations and body plan complexity. Genomics 2006, 87:783-792.

2. Aruga J: The role of Zic genes in neural development. Molec Cell Neurosci 2004, 26:205-221.

3. Merzdorf CS: Emerging roles for zic genes in early development. Devl Dynamics 2007, 236:922-940.

4. Mizugishi K, Hatayama M, Tohmonda T, Ogawa M, Inoue T, Mikoshiba K, Aruga J: Myogenic repressor I-mfa interferes with the function of of Zic family proteins. Biochem Biophysl Res Commun 2004, 320:233-240.

5. Lindgens D, Holstein TW, Technau U: Hyzic, the Hydra homolog of the zic/ odd-paired gene, is involved in the early specification of the sensory nematocytes. Development 2004, 131:191-201.

6. Srivastava M, Simakov O, Chapman J, Fahey B, Gauthier MEA, Mitros T, Richards GS, Conaco C, Dacre M, Hellsten U, Larroux C, Putnam NH, Stanke M, Adamska M, Darling A, Degnan SM, Oakley TH, Plachetzki DC, Zhai Y, Adamski M, Calcino A, Cummins SF, Goodstein DM, Harris C, Jackson DJ, Leys SP, Shu S, Woodcroft BJ, Vervoort M, Kosik KS, Manning G, Degnan BM, Rokhsar DS: The Amphimedon queenslandica genome and the evolution of animal complexity. Nature 2010, 466:720-727.

7. Benedyk MJ, Mullen JR, DiNardo S: Odd-paired: a zinc finger pair-rule protein required for the timely activation of engrailed and wingless in Drosophila embryos. Genes Dev 1994, 8:105-117.

8. Cimbora DM, Sakonju S: Drosophila midgut morphogenesis requires the function of the segmentation gene odd-paired. Dev Biol 1995, 169:580-595.

9. Skeath JB, Panganiban G, Selegue J, Carroll SB: Gene regulation in two dimensions: the proneural achaete and scute gnees are controlled by combinations of axis-patterning genes through a common intergenic control region. Genes Dev 1992, 6:2606-2619.

10. Alper $S$, Kenyon C: The zinc finger protein REF-2 functions with the Hox genes to inhibit cell fusion in the ventral epidermis of $C$. elegans. Development 2002, 129:3335-3348.

11. Bertrand $\mathrm{V}$, Holber $\mathrm{O}$ : Linking asymmetric cell division to the terminal differentiation program of postmitotic neurons in C. elegans. Dev Cell 2009, 16(4):563-575.

12. Takahashi H, Shimizu T, Aruga J: Expression pattern of the annelid Zic in embryonic development of the oligochaete Tubifex tubifex. Dev Genes Evol 2008, 218:553-560.

13. Nagai T, Aruga J, Takada S, Gunther T, Sporle R, Schughard K, Mikoshiba K: The expression of the mouse Zic1, Zic2, and Zic3 gene suggests an essential role for Zic genes in body pattern formation. Dev Biol 1997, 182:299-313.

14. Gostling NJ, Shimeld SM: Protochordate Zic genes define primitive somite compartments and highlight molecular changes underlying neural crest evolution. Evol Dev 2003, 5(2):136-144. 
15. Wada S, Saiga H: HrzicN, a new Zic family gene of ascidians, plays essential roles in the neural tube and notochord development. Development 2002, 129:5597-5608.

16. Imai KS, Satou Y, Satoh N: Multiple functions of a Zic-like gene in the differentiation of notochord, central nervous system and muscle in Ciona savignyi embryos. Development 2002, 129:2723-2732.

17. Nishida $H$, Sawada K: macho-1 encodes a localized mRNA in ascidian eggs that specifies muscle fate during embryogenesis. Nature 2001, 409:724-729.

18. Gyoja F: Expression of a muscle determinant gene, macho-1, in the anural ascidian Molgula tectiformis. Dev Genes Evol 2006, 216:285-289.

19. Ronquist F, Huelsenbeck JP: MRBAYES 3: Bayesian phylogenetic inference under mixed models. Bioinformatics 2003, 19:1572-1574.

20. Guindon S, Gascuel O: A simple, fast, and accurate algorithm to estimate large phylogenies by maximum likelihood. Syst Biol 2003, 52:696-704.

21. Meyer NP, Seaver EC: Neurogenesis in an annelid: characterization of brain neural precursors in the polychaete Capitella sp. I. Dev Biol 2009, 335:237-252.

22. Meyer NP, Boyle MJ, Martindale MQ, Seaver EC: A comprehensive fate map by intracellular injection of identified blastomeres in the marine polychaete Capitella teleta. EvoDevo 2010, 1:8.

23. Dill KK, Thamm K, Seaver EC: Characterization of twist and snail gene expression during mesoderm and nervous system development in the polychaete annelid Capitella sp. I. Dev Genes Evol 2007, 217:435-447.

24. Hernandez-Nicaise ML: Ctenophora. In Microscopic Anatomy of Invertebrates, Placaozoa, Porifera, Cnidaria and Ctenophora. Volume 2. Edited by: Herrison FW, Westfall JA. New York: Wiley-Liss; 1991:359-418.

25. Horridge GA: Presumed photoreceptive cilia in a ctenophore. Q J Micr Sci 1964, 105:311-317

26. Dunn CW, Hejnol A, Matus DQ, Pang K, Browne WE, Smith SA, Seaver E, Rouse GW, Obst M, Edgecombe GD, Sorensen MV, Haddock SHD, Schmidt Rhaesa A, Okusu A, Kristensen RM, Wheeler WC, Martindale MQ, Giribet G: Broad phylogenetic sampling improves resolution of the animal tree of life. Nature 2008, 452:745-749.

27. Hejnol A, Obst M, Stamatakis A, Ott M, Rouse GW, Edgecombe GD, Martinez P, Baguna J, Bailly X, Jondelius U, Wiens M, Werner E, Muller G, Seaver E, Wheeler WC, Martindale MQ, Giribet G, Dunn CW: Assessing the root of the bilaterian animals with scalable phylogenomic methods. Proc Royal Soc Bioll Sci 2009, 476:4261-4270.

28. Pick KS, Philippe H, Schreiber F, Erpenbeck D, Jackson DJ, Wrede P, Wiens M, Alie A, Morgenstern B, Manuel M, Worheide G: Improved phylogenomic taxon sampling noticeably affects non-bilaterian relationships. Molec Biol Evol 2010, 27:1983-87.

29. Collins AG: Evaluating multiple alternative hypotheses for the origin of Bilateria: An analysis of 18 S rRNA molecular evidence. PNAS 1998, 95:15458-15463.

30. Philippe H, Derelle R, Lopez P, Pick K, Borchiellini C, Boury-Esnault N, Vacelet J, Renard E, Houliston E, Queinnec E, Da Silva C, Winicker P, Le Guayader H, Leys S, Jackson DJ, Schreiber F, Erpenbeck D, Morgenstern B, Woerheide G: Phylogeomics revives traditional views on deep animal relationships. Curr Biol 2009, 19:706-712.

31. Srivastava M, Begovic E, Chapman J, Putnam NH, Hellsten U, Kawashima T, Kuo A, Mitros T, Salamov A, Carpenter ML, Signorovitch AY, Moreno MA, Kamm K, Grimmwood J, Schmutz J, Shapiro H, Grigoriev IV, Buss LW, Schierwater B, Dellaporta SL, Rokhsar DS: The Trichoplax genome and the nature of placozoans. Nature 2008, 454:955-960.

32. Lamar E, Kinter C, Goulding M: Identification of NKL, a novel Gli-Kruppel zinc-finger protein that promotes neural differentiation. Development 2001, 128:1335-1346.

33. Kim YS, Lewandoski M, Perantoni AO, Kurebay S, Nankanishi G, Jetten AM: Identification of Glis1, a novel Gli-related, Kruppel-like zinc finger protein containing transactivation and repressor functions. J Biol Chem 2002, 277:30901-30913.

34. Shimeld SM: $\mathrm{C}^{2} \mathrm{H} 2$ zinc finger genes of the Gli, Zic, KLF, SP, Wilms' tumour, Huckebein, Smail, Ovo, Spalt, Odd, Blimp-1, Fez and related families from Branchiostoma floridae. Dev Genes Evol 2008, 218:639-649.

35. Marlow $\mathrm{HQ}$, Srivastava $\mathrm{M}$, Matus $\mathrm{DQ}$, Rokhsar $\mathrm{D}$, Martindale MQ: Anatomy and development of the nervous system of Nematostella vectensis, and Anthozoan Cnidarian. Dev Neurobiol 2009, 69:235-254.
36. Materna SC, Howard-Ashby M, Gray RF, Davidson EH: The $\mathrm{C} 2 \mathrm{H} 2$ zinc finger genes of Strongylocentrotus purpuratus and their expression in embryonic development. Dev Biol 2006, 300:108-120.

37. Nusslein-Volhard C, Wieschaus E: Mutations affecting segment number and polarity in Drosophila. Nature 1980, 287:795-801.

38. Edgar RC: MUSCLE: multiple sequence alignment with high accuracy and high throughput. Nucleic Acids Res 2004, 32:1792-1797.

39. Abascal F, Zardoya R, Posada D: ProtTest: selection of best-fit models of protein evolution. Bioinformatics 2005, 21:2104-2105.

40. Pang K, Martindale MQ: Developmental expression of homeobox genes in the ctenophore Mnemiopsis leidyi. Dev Genes Evol 2008, 18:307-319.

doi:10.1186/2041-9139-1-12

Cite this article as: Layden et al: Expression and phylogenetic analysis of the zic gene family in the evolution and development of metazoans. EvoDevo 2010 1:12.

\section{Submit your next manuscript to BioMed Central and take full advantage of:}

- Convenient online submission

- Thorough peer review

- No space constraints or color figure charges

- Immediate publication on acceptance

- Inclusion in PubMed, CAS, Scopus and Google Scholar

- Research which is freely available for redistribution

Submit your manuscript at www.biomedcentral.com/submit 